\title{
Aprender: la ventaja COMPETITIVA más SOSTENIBLE EN EL TIEMPO
}

\author{
Julio Andrés Palomino Silva \\ Docente-Facultad de Ciencias Contables
}

\begin{abstract}
RESUMEN
Durante estas últimas décadas el entorno empresarial ha cambiado radicalmente. Y dentro de este entorno el saber, los conocimientos no solo han adquirido una importancia vital, sino que su propio significado ha variado, transformado la sociedad y la economia.

El crear en nuestra organización una cultura de aprendizaje permanente es un recurso económico básico, el obtener una ventaja competitiva a través del aprendizaje permanente en la que las personas se conviertan en el centro de los modelos de enseñanza empresarial es un reto que hoy tiene toda organización.
\end{abstract}

Palabras clave: Ventajas competitivas, capacidad empresarial, herramientas de gestión.

\section{IN'TRODUCCIÓN}

Peter Drucker (1993) define las organizaciones: "Como un grupo humano de especialistas que trabajan unidos en la consecución de una tarea común". Considera que una organización, comotoda creación humana, está hecha para existir durante un periodo considerable de tiempo, que siempre se especializa en una tarea concreta que la defina y que su eficiencia viene dada por el grado de concentración en esta tarea. Para Henry Minzberg: "Organización significa acción colectiva para realizar una misión común, una manera elegante de decir que un puñado de gente se ha reunido bajo un nombre común para producir algún producto o servicio. Tom Peters, el "enfant terrible" del management, plantea -como es habitual en él-una definición mas controvertida. Para él: "Las organizaciones son grupos algo duraderos de individuos que realizan ciertas actividades de las que, por lo menos algunas de ellas, se dirigen a la consecución de fines comunes a todos".

Todas las definiciones que acabamos de citar son discutibles en muchos de sus puntos, pero lo que si es cierto es que en el origen de todas las organizaciones se encuentran una o varias personas que han ido madurando una idea con el paso del tiempo, hasta que un día consideran, llegado el momento, ponerla en práctica. Los directivos con espíritu emprendedor perciben oportunidades y poseen la habilidad de combinar diferentes recursos para producir nuevos productos o servicios. Todoellotienelugar a menudoen un entorno 
político-social y económico, en donde los incentivos de mercado y los recursos económicos no existen o tienen un coste extremadamente elevado y en donde ni la cultura, ni los valores, ni la legislación favorecen este tipo de iniciativas.

\section{VENTAJA COMPETITIVA Y CADENA DE VALOR}

Este difícil proceso de transformación de viejos y nuevos proyectos en realidades requiere el desarrollo de una estructura organizativa que coordine los diferentes elementos para obtener los objetivos previstos. Esta organización la definimos como un sistema que recibe, transforma y factura inputs (véase Figura 1).

Todo este proceso tiene por objetivo añadir valor a nuestras organizaciones, para ello se requiere adquirir y consumir recursos (dinero, trabajo, materias primas, equipos, edificios y tecnología). Este proceso se efectúa a partir de un conjunto de subsistemas (cadena de valor) que añaden valor al producto o servicio de sus actividades (diseño, aprovisionamiento, producción, marketing, ventas, transporte $\mathrm{y}$ apoyo de sus productos).

El conjunto de subsistemas contribuye al desarrollo de la ventaja competitiva a través de los costes (utilización eficiente de la fuerza de ventas, sistema de distribución de bajo coste, procesos de ensamblaje eficiente), que nos permite reducir el precio de los recursos y la cantidad de recursos utilizados y de la diferenciación, a través de las actividades quegeneran productos diferentes o mejores que sus competidores (obtención de materias primas de alta calidad, diseño, sistema ágil de entrada de pedidos). El objetivo de toda estrategia genérica de nuestras organizaciones debe ser crear valor. El margen no es nada más ni nada menos que la diferencia entre el valor generado y los costos en que incurrimos para generarlo.

Las ventajas competitivas surgen de los recursos y capacidades que desarrollan nuestras organizaciones para sobrevivir y fortalecer nuestras posiciones respecto a la competencia. Los recursos son los factores disponibles controlados por nuestras organizaciones. Los recursos tangibles (patentes, licencias, activos financieros, activos físicos, capital humano) los podemosadquirirsino disponemos de ellos; el dilema que se nos presenta en algunos casos se refiere al coste de oportunidad de generarlos en nuestras organizaciones o adquirirlos en el mercado. Los recursos intangibles (los conocimientos, la imagen de marca, la lealtad de los empleados) se generan internamente. Las capacidades desarrollan los recursos, generalmente, en combinación, utilizando procesos organizativos para conseguir un fin concreto (buen servicio, respuesta rápida a las tendencias del mercado, alta calidad e innovación). Las actividades se basan en el desarrollo, transporte e intercambio de información a través del capital humano en nuestras organizaciones; son producto del aprendizaje colectivo y están impregnadas en las rutinas, procesos y la cultura de organización. El valor de un recurso o una capacidad vienedefinido por la combinación de tres variables: su escasez, su demanda y su apropiabilidad. 


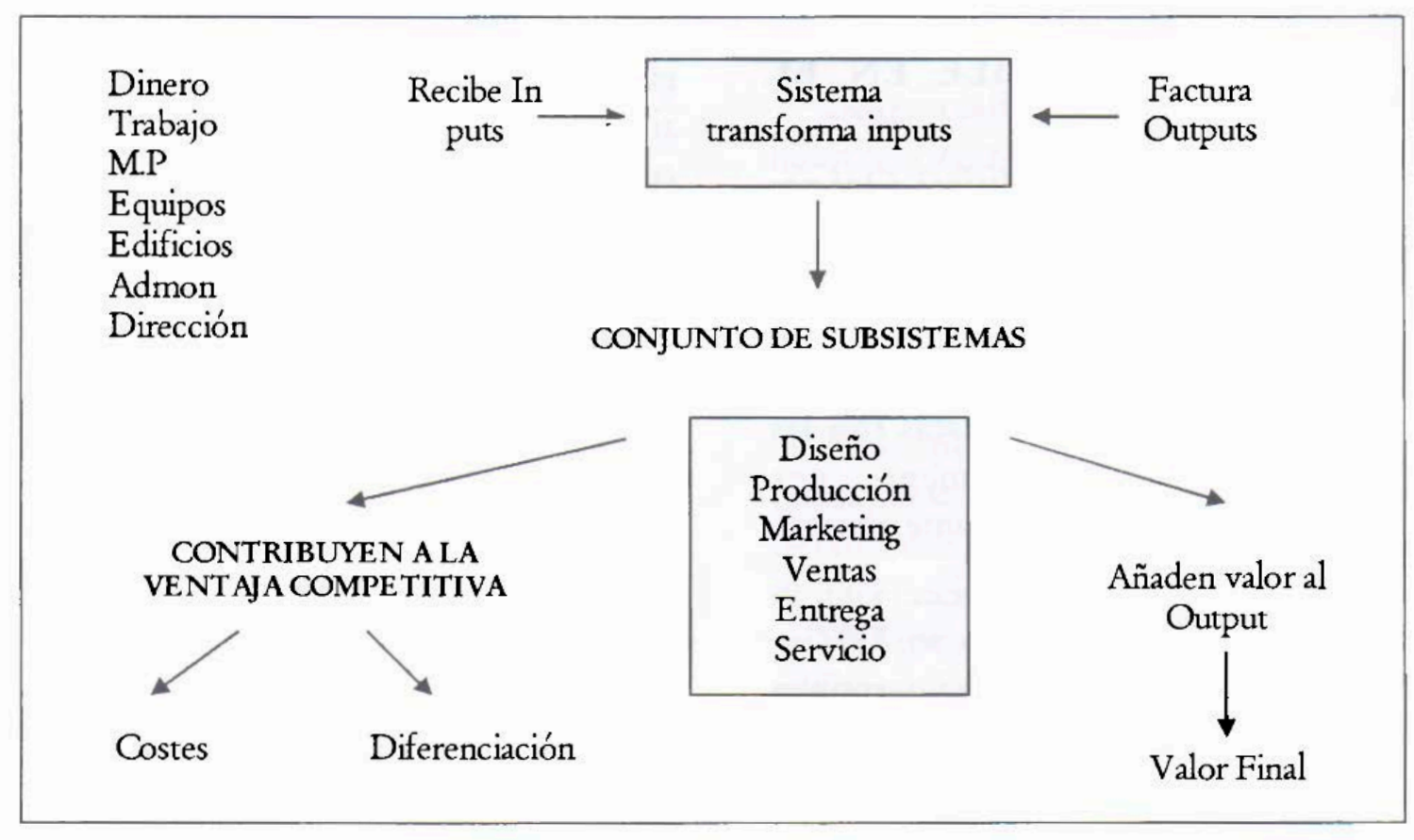

Figura 1

El concepto de ventaja competitiva posee un gran paralelismo con el concepto darwiniano de la supervivencia de la especies. Así como las especies desar rollan capacidades para adaptarse a entornos cambiantes y sobrevivir en hábitats donde los recursos son escasos y la lucha por los mismos deviene implacable, así nuestras organizaciones deben desarrollar sus propias capacidades para alcanzar sus objetivos a corto, medio y largo plazo. La ventaja competitiva es la herramienta imprescindible para crear valor, entendiéndose por valor la cantidad de dinero que nuestros clientes están dispuestos a pagar por nuestros productos de las diferentes actividades de nuestras organizaciones, forma parte del sistema de valor del sector en que se halla. (Véase Figura 2: Un ejemplo del sector Industrial.)

Este sistema incluye la cadena de valor de los proveedores, de los canales de distribución $\mathrm{y}$, finalmente, los productos acaban formando parte de la cadena de valor de los clientes; la mejor manera de consolidar y fortalecer la posición de nuestra organización es ayudar a nuestros clientes y proveedores a mejorar su propia cadena de valor.

\section{EMPRESA DE UN SOLO SECTOR INDUSTRIAL}

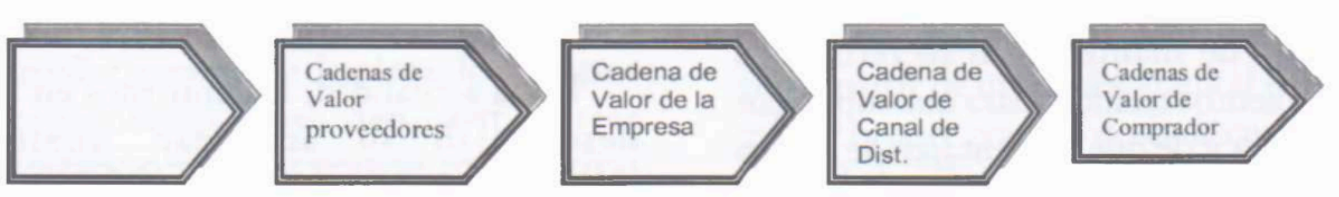

Figura 2 


\section{LA VENTAJA COMPETITIVA HA DE SER SOSTENIBLE EN EL TIEMPO}

¿Por qué tenemos beneficios? ¿Qué nos hace diferenciarnos de la competencia? ¿Somos capaces de sostener la ventaja que hemos obtenido a partir del desarrollo de capacidades únicas? ¿Esta ventaja es sostenible en el tiempo? Estas son las preguntas que habitualmente nos planteamos o deberíamos plantearnos en nuestras organizaciones.

Tradicionalmente (Ghemawatt: 1987) se ha considerado que las ventajas susceptibles de sostenerse en el tiempo son básicamente las que hacen referencia a:

- El tamaño de la organización en el mercado.

- El acceso a los recursos y la clientela.

- Las restricciones en las opciones de la competencia.

Las ventajas relacionadas con el tamaño tienen un valor real, si en el sector en que nos hallamos se pueden dar economías inherentes al tamaño de la organización. El tamaño proporciona ventajas porque los mercados son finitos. Nuestros competidorespueden resignarsea continuar siendo pequeños, conscientes de que es mejor ser pequeño en un mercado rentable que generar una oferta excesiva que acabe siendo ruinosa para todos.

Un mejor acceso a los recursos técnicos y los factores de producción se debe, en general, a las ventajas generadas por la experiencia y el tamaño y al hecho de que la oferta es limitada. El problema surge de la dificultad de mantener en secreto estos conocimientos técnicos para que supongan una ventaja y de que las ventajas de acceso a los factores dependen de su disponibilidad y precio. Basar la ventaja competitiva en el hecho de poder adquirir los conocimientos en mejores condiciones o en que sigan siendo aplicables a largo plazo puede convertirse en un error de graves consecuencias.

El acceso a los mercados viene marcado por su sensibilidad a las preferencias de los consumidores. La competencia puede verse mermada en sus opciones por aspectos políticos- legales (patentes), reacciones defensivas debido a falta de recursos económicos y lentitud en las respuestas. Tradicionalmente, los recursos y capacidades son duraderos $\mathrm{y}$ específicos, también los sectores que evolucionan gradualmente ofrecen motores, posibilidades de mantener las ventajas competitivas que los que son sacudidos por cambios drásticos en la tecnología y en la demanda.

Este análisis tradicional plantea múltiples interrogantes en la actualidad. Lo más probable es que nuestros competidores estén intentando implantar estrategias similares a las nuestras, copiar nuestra tecnología o imitar nuestras innovaciones. La realidad nos demuestra que la competencia reacciona rápidamente ante cualquier cambio, tanto en la innovación de productos como de las operaciones. Las imitaciones que llevan a cabo nuestros competidores son menos costosas y mucho más rápidas que nuestra innovaciones y la curva de aprendizaje de los procesos productivos es, también, fácilmente copiable. Porotro lado, cada vezes más difícil encontrar entornos con las características que se planteaban tradicionalmente.

En la actualidad, los entornos en que desarrollan su actividad nuestras organizaciones se caracterizan por su: 
- Incertidumbre acerca de los parámetros económicos, legales, políticos y tecnologías de las preferencias de los consumidores y del comportamiento de la competencia.

- Complejidad de las causas interrelacionadas que forman el entorno de la organización. Los grupos de interés externos desempeñan cada vez un papel más importante en la gestión de nuestras organizaciones. Los efectos positivos o negativos que nuestras decisiones tienen sobre ellos puede generar, a su vez, una reacción que nos puede afectar positiva $o$ negativamente.

- Conflictos intraorganizacionales entre los que forman las decisiones y los afectados por las mismas. Los grupos de interés internos desempeñan, a su vez, un papel más activo cada día. Tanto nuestros accionistas como nuestros empleados no se limitan únicamente, comotradicionalmenteera el caso, a exigir más flujoseconómicos por su aportación en capital y en trabajo; en la actualidad, en muchos casos, exigen el derecho a opinar y controlar la gestión de los altos directivos sobre temas como la calidad de los productos, las condiciones de trabajo, el respeto al medio ambiente, la seguridad, lapublicidad,ladiscriminación y el papel de la organización en la comunidad.

Todo ello nos lleva a cuestionar la sostenibilidad en el tiempo de las ventas competitivas tradicionales, y nos plantea de nuevo el reto constante de todo empresario y directivo con vocación de futuro a identificar, desarrollar, proteger y desplegar los recursos y capacidades que den a nuestras organizaciones nuevas ventajas competitivas sostenibles en el tiempo.

\section{ENDOGAMIA EMPRESARIAL}

Desgraciadamente, en muchas de nuestras organizaciones funcionamos a menudo según criterios establecidos en sus inicios, que se han ido utilizando sin ningún espíritu crítico que cuestione su solidez o eficacia, y que acaban generando unos modelos de dirección endogámicos. Estos modelos aplican herramientas de gestión (presupuestos, planificación, sistemas de recompensas, control, socialización, sistemas de información) que acaban convirtiéndose en códigos que nos ayudan a gestionar nuestras organizaciones de una manera rutinaria. Con el tiempo, este proceso nos lleva a dogmatizar ciertas creencias y a codificar como infaliblesnuestros criterios de gestión. Este tipo de codificación nos condiciona a no preocuparnos por saber lo que no sabemos y a no plantearnos por qué creemos lo que creemos. Consecuencia de todoello esel desarrollo lento pero imparable de una cultura empresarial endogámica, abono perfecto para las semillas de la autodestrucción.

Esta creencia en la infalibilidad de nuestros modelos degestión no nos permiteapreciar los cambios que se producen en nuestro entorno y los nuevos parámetros que marcan el quehacer futuro de la gestión empresarial. Nuestras organizaciones deben dejar de ser las famosas cajas negras con que se definía tradicionalmente a las organizaciones empresariales para transformarse en organizaciones transparentes, capaces de dar y recibir información. Todo proceso de cambio exige cuestionar el marco genético de la organización. Este cambio únicamente se puede lograr a partir de un cambio radical de actitud que incorpore grandes dosis de curiosidad y humildad, y desarrolle una total disposición a olvidar y, sobre todo, a aprender. 


\section{LA REVOLUCIÓN DEL SABER}

Desde la antigüedad, el saber o conocimiento estuvo íntimamente relacionado con el ser; el conocimiento permitía a los ciudadanos saber qué decir y cómo decirlo. Para Platón, la función del saber tenía por finalidad el autoconocimiento y el desarrollo de la persona. En oriente, las teorías sobre el saber coincidían en su significado con las teorías de la Grecia clásica y el Trivium que se consolidó en la Edad Media; el conocimiento tenía por objetivo el desar rollo del ser. La capacidad de hacer estaba reservada al arte, (techne, en griego); las artes se aprendían a partir de la experiencia.

La tecnología surge de la combinación entre el arte manual (techne) y el saber organizado (logos) que se desarrolla, sobre todo, a partir de los trabajos de recopilación del saber iniciado por los pensadores franceses del siglo XVII. Esta combinación, que en la actualidad denominamos tecnología, ha marcado el devenir de la humanidad en los últimos doscientos años. El saber que tradicionalmente se venía aplicando al ser, se empieza a aplicar al hacer. En una primera etapa -la revolución industrialel saber se aplicó a las herramientas, procesos y productos. En una segunda etapa, a partir de la organización científica del trabajo de Taylor, el conocimiento se aplicó, por primera vez, al estudio del trabajo humano. Todo trabajo manual puede ser analizado y organizado a través de la aplicación del conocimiento. En la última etapa, el conocimiento se acaba aplicando el conocimiento. El objetivo fundamental consiste en aportar conocimiento para averiguar en qué forma el saber existente puede aplicarse a producir resultados. Esta última etapa es la que Drucker denomina la revolución de la gestión. A partir de aquí el gerente deja de ser únicamente el responsable de sus subordinados y del rendimiento de los mismos para asumir el papel de "responsable dela aplicación y rendimiento del saber". El saber se convierte en el recurso económico básico, desbancando del primer lugar a la troika tradicional: capital, tierra, trabajo.

En este nuevo contexto, en el que el saber se convierte en protagonista principal del quehacer económico y en el que, como hemos visto anteriormente, la vida media delos conocimientos y de las innovaciones tecnológicas disminuye constantemente, la flexibilidad y el saber (junto con la capacidad de aprender) se han convertido en los nuevos recursos sostenibles, en las nuevas ventajas competitivas. Las organizaciones que quieren mantener e incrementar su nivel de competitividad deberán transfor marse en organizaciones que aprenden, en centros de aprendizajes permanentes en el que las personas se convierten en el centro de los modelos de enseñanza empresarial.

Para saber se requiere la capacidad de aprender, de reflexionar sobre nuestros actos, de combinar el trabajo y el aprendizaje. Se requiere lo que Aubrey y Cohen (1995) definen como la sabiduría: "un recurso vital y en buena parte desaprovechado, una forma de capital humano adquirido por la actividad propia, acumulando mediante la experiencia y aplicado aprendiendo en el trabajo diario".

Las organizaciones que se plantean la necesidad de convertir el aprendizaje en una estrategia per manente activan este concepto convirtiéndolo en un punto de referencia, 
en una cultura, en una praxis diaria de ayuda a las personas a aprender que incrementa el desarrollo personal y profesional de todos los miembros de la organización.

\section{APRENDER, LA VENTAJA MÁS SOSTENIBLE EN EL TIEMPO}

Hemos visto que muchas de las ventajas competitivas tradicionales adolecían de un grave defecto: eran difícilmente sostenibles en el tiempo. A veces las causas eran intrínsecas al sector -en el sector financiero la innovación de productos es una ventaja competitiva difícilmente sostenible: los nuevos productos financieros son fácilmente copiables por la competencia-. En otros casos, la capacidad de nuestros competidores para copiar nuestros productos y procesos productivos les permitía disponer de productos de una calidad similar, pero con un coste de desarrollo inferior al nuestro. Finalmente, la causa más importante es nuestra incapacidad, producto de nuestra endogamia empresarial, para desarrollar nuevas ventajas competitivas sostenibles en el tiempo a partir de nuestros recursos y capacidades.

Ello no es óbice para que existan ventajas competitivas más sostenibles que otras, como es el caso de las organizaciones que han sido capaces de desarrollar una imagen de marca o una cultura de servicio al cliente. En la actualidad, dentro de la cadena de valor de muchos sectores de nuestra economía, las ventajas competitivas se concentran progresivamente en las actividades que añaden valor a partir del momentode la venta del producto o servicio. Desde los servicios de formación, preparación, instalación, reparación, mantenimiento y sustitución de múltiples productos de consumo -automóviles, computadoras, sistemas de riego, aparatos de limpieza industrial, etc., hasta las bolsas de trabajo y las actividades académicas de las asociaciones de antiguos alumnos de las escuelas empresariales-. Todas ellas tienen como objetivo añadir valor al producto o servicio, aportando algo más y mejor que la competencia. Sin embargo, el desarrollo y mantenimiento de la capacidad de aprender en nuestras organizaciones supone una ventaja competitiva que intrínsecamente garantiza su sostenibilidad en el tiempo.

La verdadera capacidad de aprender tiene sus orígenes en la motivación interna de cada miembro de la organización a desarrollarse personal y profesionalmente para bien propio y de la organización. Cuando la motivación depende de factores externos -salarios, planes de formación, jubilaciones generosas, promociones- la capacidad de aprender está condicionada por las decisiones de los altos directivos respecto a estos factores. El crear en nuestra organización estacultura de aprendizaje permanentemente, a través de la motivación interna, convierte esta ventaja competitiva en algo sostenible. En este tipo de organizaciones, los profesionales cuestionan, a menudo, los planes tradicionales de for mación que reciben, en muchos casos, como elementos externos de motivación. En estos planes, la organización fija los objetivos, los resultados deben ser medibles, y los conocimientos impartidos deben ser aplicables a una sola actividad profesional. Este planteamientochoca frontalmente con un modelo de organización en aprendizaje permanente, en que la formación debe plantear objetivos a largo plazo, tanto personales como profesionales, y los conocimientos deben estar orientados a actividades múltiples y abiertas. 
Si somos capaces de desarrollar en nuestros organizaciones esta cultura de aprendizaje permanente, a través de motivación interna de sus profesionales, la pregunta que a continuación nos corresponde dilucidar es si será o no sostenible en el tiempo. Tradicionalmente se ha considerado que para que una ventaja competitiva sea sostenible en el tiempo, los recursos y capacidades que la sustentan deben reunir las siguientes características:

- Inimitabilidad: ¿Es el recurso o la capacidad difícil de imitar? Si son fácilmente imitables, laventaja competida será difícilmente sostenible en el tiempo.

- Durabilidad: ¿Cuánto tiempo pueden mantener la ventaja competitiva? La mayoría de recursos y capacidades tienen una vida limitada, sobre todo, en sectores de un alto dinamismo en donde su valor se deprecia rápidamente.

- Apropiabilidad: ¿Ha sido la organización la receptora del valor generado por el recurso o la capacidad? Los diferentes grupos de interés de la organización pueden estar interesados en apropiarse de parte del valor generado.

- Sustituibilidad: ¿Es el recurso o la capacidad sustituible por otro?

- Superioridad competitiva: ¿Hay algún recurso o capacidad que genere una ventaja competitiva superior al aprendizaje permanente?

La respuesta a la pregunta planteada anteriormente: será o no sostenible en el tiempo, parece evidente. Las organizaciones quese transfor man en centros de aprendizaje permanentes están creando una ventaja competitiva que reúne las características necesarias para garantizar su sostenibilidad en el tiempo. La capacidad de aprendizaje permanente requiere no solo una voluntad firme, sino un periodo de desarrollo largoy costoso. Su durabilidad dependerá, en parte, de la capacidad de reacción de los competidores, pero su valor difícilmente se depreciará mientras el espíritu de aprendizaje se mantenga vivo. El valor generado beneficiará a las personas y a la organización, como el conjunto de estas personas que trabaja unido en la consecución de un objetivo común. El saber $y$, finalmente, su superioridad competitiva es única en una sociedad en la que, como hemos visto, el saber se ha convertido en el recurso económico que viene marcado por la progresiva globalización de mercados y productos, la revolución de los sistemas de comunicación, la rápida rotación de conocimientos en la mayoría de sectores, pero, sobre todo, en los de mayor futuro, y la consiguiente obsolescencia de muchas de las ventajas competitivas tradicionales.

\section{REFERENCIAS}

1 Argyrys, Chpis. Good Comunicación that Blocks Learning. Harvard Business Review, junio-agosto, 1994.

2 Aubrey, R. y Cohen P. La organización en aprendizaje permamente. Editorial Deusto, Bilbao, 1995.

3 Collins, D.J., Montgomery.C.H. Competing on Resources; Strategy in the 1990's. Harvard Business Review, julio-agosto, 1995.

4 Drucker, P. Post-Capitalist Society. ButterWorth - Heinemann Ltd., Oxford, 1993.

5 Ghemawat, P. La ventana competiti ra ba de ser sostenible. Harvard Deusto Busines Review, 2. ${ }^{\circ}$ trimestre, 1987.

6 Goldrat, E. No es cuestión de suerte. Díaz de Santos, Madrid, 1995.

7 Minyzberg, H. Mintzbergyladirección Díaz de Santos, Madrid, 1991.

8 Peter, Tom. Nuezas organizaciones en tiempos de caos. Deusto, Bilbao, 1994. 